\title{
Anforderungen an die Hygiene bei der Aufbereitung von Medizinprodukten
}

Eine ordnungsgemäße Aufbereitung wird laut Medizinprodukte-Betreiberverordnung (§ 4 Absatz 2) vermutet, wenn die gemeinsame Empfehlung der Kommission für Krankenhaushygiene und Infektionsprävention (KRINKO) beim Robert Koch-Institut und des Bundesinstitutes für Arzneimittel und Medizinprodukte (BfArM) zu den „Anforderungen an die Hygiene bei der Aufbereitung von Medizinprodukten " beachtet wird. Diese Vermutung wird seit Sommer 2011 auch explizit im \$23 Absatz 3 des IfSG für alle veröffentlichten Empfehlungen der Kommission für Krankenhaushygiene und Infektionsprävention beim Robert Koch-Institut ausgesprochen und findet sich auch in Landesverordnungen wieder.

Die sogenannte KRINKO-BfArM-Empfehlung stammte bereits aus dem Jahre 2001 und bedurfte einer Aktualisierung. Zum einen wurden zwischenzeitlich weitere Empfehlungen und Kommentare zu speziellen Bereichen der Aufbereitung von der KRINKO und dem BfArM veröffentlicht. Zum anderen sollten natürlich auch weitere aktuelle Veröffentlichungen, wie z. B. der Erfahrungsbericht zur Aufbereitung von Medizinprodukten in Deutschland des BMG aus dem Jahre 2008, europäische und nationale Standards bzw. Normen, Originalveröffentlichungen zu Aspekten der Aufbereitung sowie Leitlinien und Empfehlungen von Fachgesellschaften bei der Aktualisierung berücksichtigt werden. Auf dieser Grundlage wurde ein neuer Entwurf der KRINKO-BfArM-Empfehlung in einer interdisziplinären Arbeitsgruppe erstellt und in der KRINKO unter Beteiligung des BfArM diskutiert. Daraufhin erfolgte das für Empfehlungen der KRINKO vorgesehene Anhörungsverfahren mit Beteiligung der Länder und von Verbänden (u.a. AWMF, BÄK, BZÄK, DGKH, DGSV, DIN, DKG, DPR, KBV, PKV, VDI, VHD, ZLG), die anschließende Beratung der eingegangenen Kommentare in der Kommission unter Beteiligung des BfArM und schließlich die Verabschiedung der aktualisierten Empfehlung.

Die zentrale Empfehlung „Anforderungen an die Hygiene bei der Aufbereitung von Medizinprodukten“ ist mit Rücksicht auf die erfolgreiche Anwendung in der Ver- gangenheit in ihrem prinzipiellen Aufbau größtenteils gleich geblieben und wurde jeweils an den entsprechenden Stellen aktualisiert bzw. um einzelne Aspekte (wie z. B. konkretisierende Angaben zu manuellen Schritten der Aufbereitung, Zwischenspülung, Prüfung der viruziden Wirksamkeit eines Desinfektionsmittels, Heißluftsterilisation, Rückverfolgbarkeit) ergänzt.

Neu sind insgesamt acht mitgeltende Anlagen und weitere Anhänge zur Konkretisierung von einzelnen Aspekten der zentralen Empfehlung:

- Anlage 1 Zum Begriff „geeignete validierte Verfahren"

- Anlage $2 \mathrm{Zu}$ Abschnitt 2.2.3 „Prüfung der technisch-funktionellen Sicherheit ${ }^{*}$

- Anlage 3 Inbetriebnahme und Betrieb von Reinigungs-Desinfektionsgeräten (RDG) zur Aufbereitung von Medizinprodukten (Checkliste)

- Anlage 4 Inbetriebnahme und Betrieb von Kleinsterilisatoren zur Aufbereitung von Medizinprodukten (Checkliste)

- Anlage 5 Übersicht über Anforderungen an Aufbereitungseinheiten für Medizinprodukte

- Anlage 6 Sachkenntnis des Personals

- Anlage 7 Maßnahmen zur Minimierung des Risikos einer Übertragung der CJK/ vCJK durch Medizinprodukte

- Anhang 1 zur Anlage 7 Hinweis auf den Endoskop-Pool an der Universitätsmedizin Göttingen

- Anlage 8 Anforderungen an die Hygiene bei der Aufbereitung flexibler Endoskope und endoskopischen Zusatzinstrumentariums

- Anhang 6 zur Anlage 8 Zur Aufbereitung flexibler Zystoskope und Bronchoskope

- Anhang 7 zur Anlage 8 Aufbereitung von Ultraschallsonden zur Anwendung in der Gynäkologie

- Anhang 8 zur Anlage 8 Aufbereitung von Ultraschallsonden mit Schleimhautkontakt

Die oben genannten Empfehlungen gelten grundsätzlich unabhängig vom Ort der Durchführung der Aufbereitung sowohl im ambulanten und stationären Sektor. Entscheidend für Art und Umfang der Maßnahmen sind die Komplexität des Medizin- produktes sowie die vorausgehende und die der Aufbereitung folgende Anwendung. Die Veröffentlichung im Bundesgesundheitsblatt ist Anfang Oktober 2012 erfolgt und ersetzt die entsprechende Empfehlung aus dem Jahr 2001.

Die Empfehlung und eine Musterpräsentation zur Einführung in die Thematik ist auf den Internetseiten des RKI abrufbar:

www.rki.de > Infektions- und

Krankenhaushygiene $>$ Empfehlungen der Kommission für Krankenhaushygiene und Infektionsprävention > Reinigung, Desinfektion, Sterilisation > Anforderungen an die Hygiene bei der Aufbereitung von Medizinprodukten

Marc Thanheiser Fachgebiet Angewandte Infektions- und Krankenhaushygiene Robert Koch-Institut Berlin 